\title{
The Lats2 tumor suppressor augments p53-mediated apoptosis by promoting the nuclear proapoptotic function of ASPP1
}

\author{
Yael Aylon, ${ }^{1}$ Yaara Ofir-Rosenfeld, ${ }^{2}$ Norikazu Yabuta, ${ }^{3}$ Eleonora Lapi, ${ }^{4}$ Hiroshi Nojima, ${ }^{3}$ Xin Lu, ${ }^{4}$ \\ and Moshe Oren ${ }^{1,5}$ \\ ${ }^{1}$ Department of Molecular Cell Biology, The Weizmann Institute of Science, Rehovot 76100 , Israel; ${ }^{2}$ Hutchison/MRC Research \\ Centre, Cambridge CB2, United Kingdom; ${ }^{3}$ Department of Molecular Genetics, Research Institute for Microbial Diseases, Osaka \\ University, Osaka 565-0871, Japan; ${ }^{4}$ Ludwig Institute for Cancer Research, Nuffield Department of Clinical Medicine, \\ University of Oxford, Oxford OX3 7DQ, United Kingdom
}

Apoptosis is an important mechanism to eliminate potentially tumorigenic cells. The tumor suppressor p53 plays a pivotal role in this process. Many tumors harbor mutant p53, but others evade its tumor-suppressive effects by altering the expression of proteins that regulate the 553 pathway. ASPP1 (apoptosis-stimulating protein of p53-1) is a key mediator of the nuclear p53 apoptotic response. Under basal conditions, ASPP1 is cytoplasmic. We report that, in response to oncogenic stress, the tumor suppressor Lats2 (large tumor suppressor 2) phosphorylates ASPP1 and drives its translocation into the nucleus. Together, Lats2 and ASPP1 shunt p53 to proapoptotic promoters and promote the death of polyploid cells. These effects are overridden by the Yap1 (Yes-associated protein 1) oncoprotein, which disrupts Lats2-ASPP1 binding and antagonizes the tumor-suppressing function of the Lats2/ASPP1/p53 axis.

[Keywords: ASPP1; Yap1; p53; Lats2; apoptosis; Hippo Pathway]

Supplemental material is available at http://www.genesdev.org.

Received June 1, 2010; revised version accepted September 9, 2010.

The human Lats2 (large tumor suppressor 2) gene maps to the 13q11-12 chromosomal region, a common site of loss of heterozygosity (Chen et al. 2005). Lats2 is normally largely a centrosomal protein. However, upon mitotic or oncogenic stress, Lats2 departs from the centrosome and translocates to the nucleus (Aylon et al. 2006). Nuclear Lats 2 activates p53, which is critical for the maintenance of proper chromosome number in the face of insults to the mitotic apparatus (Aylon et al. 2006). Within this process is embedded a positive feedback loop, since the transcription of the Lats2 gene is positively regulated by p53, leading to a gradual and continuous increase in nuclear Lats2 protein levels (Aylon et al. 2006).

ASPP1 (apoptosis-stimulating protein of $\mathrm{p} 53-1$ ) is a member of the ASPP family of p53-binding proteins (Sullivan and Lu 2007). The ASPP C terminus has numerous binding partners besides p53, including p73 and p63 (Gorina and Pavletich 1996) and RelA/p65 (Yang et al. 1999). It is thought that ASPP1 and ASPP2 facilitate apoptosis primarily by stimulating the binding of p53 to

${ }^{5}$ Corresponding author.

E-MAIL moshe.oren@weizmann.ac.il; FAX 972-8-9346004.

Article is online at http://www.genesdev.org/cgi/doi/10.1101/gad.1954410. proapoptotic promoters (Samuels-Lev et al. 2001). The precise manner of regulation of p53 transcription by ASPP1, however, remains unclear. This enigma is exacerbated by the fact that ASPP1 has been shown to reside primarily in the cytoplasm (Thornton et al. 2006), away from p53 target genes.

Yap1 (Yes-associated protein 1) was reported to bind both Lats2 (Kawahara et al. 2008) and ASPP2 (Espanel and Sudol 2001). Yap1 is highly expressed in a wide spectrum of human cancer cell lines and various primary tumors (Dong et al. 2007; Overholtzer et al. 2006; Steinhardt et al. 2008). Moreover, overexpression of Yap1 in nontransformed mammary epithelial cells induces epithelialto-mesenchymal transition, suppression of apoptosis, and anchorage-independent growth (Overholtzer et al. 2006). Although these data imply an oncogenic role, Yap1 has also been shown to possess proapoptotic activities (Strano et al. 2001; Levy et al. 2007).

Damage-induced apoptosis is a key feature of tumor suppression, in which p53 plays a major role. The potency of p53 as a tumor suppressor is demonstrated by its high mutation frequency in human cancers (Wang and El-Deiry 2008). Yet, many tumors manage to retain wild-type p53. In these instances, although p53 is intact, its tumor 
suppressor function is most likely compromised by genetic or epigenetic alterations in its upstream regulators (including also many of its activating partners) as well as in its downstream effectors.

In this study, we show that Lats2 and ASPP1 act together to instigate p53's proapoptotic transcription program, and explore the underlying molecular mechanisms. This Lats2-ASPP1-p53 axis is important to eliminate potentially dangerous genomically unstable cells that result from oncogene activation. Conversely, high levels of the putative oncoprotein Yapl override this protective mechanism in a manner that might then permit tumor progression. Together with the data reported in the accompanying study by Vigneron et al. (2010) in this issue of Genes \& Development, our findings delineate an intricate set of cross-regulatory interactions between Yap1, Lats2, and ASPP1 likely to play an important role in cell fate determination and cancer.

\section{Results}

Oncogenic stress induces Lats2-dependent nuclear translocation of ASPP1

Under basal conditions, ASPP1 is primarily in the cytoplasm (Fig. 1A, top panels), as reported previously (Thornton

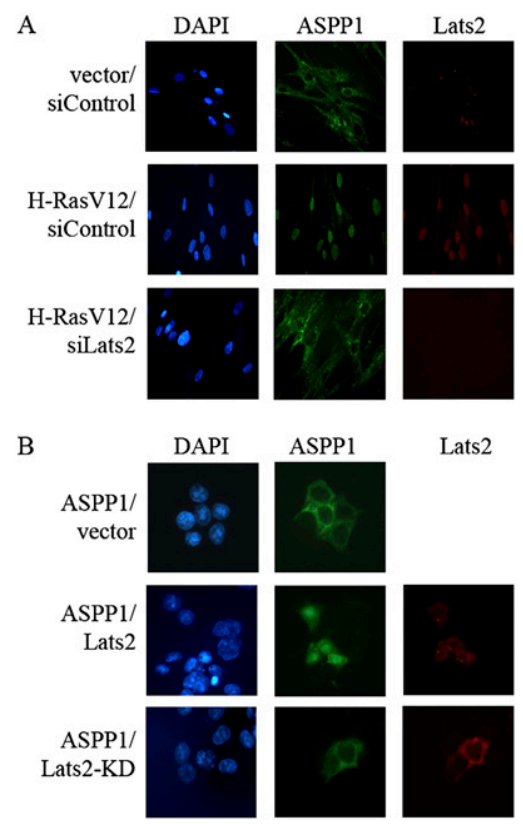

Figure 1. ASPP1 is translocated from the cytoplasm to the nucleus in a Lats2-dependent manner. (A) WI-38 cells were retrovirally infected with $\mathrm{H}$-RasV12 or empty vector only. Two days after infection, cells were transfected with Lats2 siRNA (siLats2) or control siRNA (siControl). Three days after infection, cells were fixed and immunostained with the LA2 antibody (Toji et al. 2004) to visualize endogenous Lats2, and with anti-ASPP1 antibodies. Nuclear DNA was visualized by DAPI staining. (B) HCT 116 cells were transiently transfected with V5-tagged ASPP1 together with either empty vector, kinase-active myc-tagged Lats2 (Lats2), or kinase-dead myc-tagged Lats2 (Lats2-KD). ASPP1 and Lats2 were detected with anti-V5 and anti-Lats2 antibodies, respectively. Nuclear DNA was visualized by DAPI staining. et al. 2006; Vigneron et al. 2010). However, oncogenic H-RasV12 caused significant ASPP1 accumulation in the nucleus (Fig. 1A, middle panels), reminiscent of the translocation of Lats 2 from the centrosome to the nucleus following H-RasV12 expression (Aylon et al. 2009). Remarkably, the nuclear accumulation of ASPP1 was dependent on Lats2, since, in cells with diminished levels of Lats2, ASPP1 remained cytoplasmic despite H-RasV12 expression (Fig. 1A, bottom panels; Supplemental Fig. S1A,B, protein and RNA knockdown validation). Similar results were obtained with a different siRNA targeting the Lats2 mRNA 3' untranslated region (UTR) (Supplemental Fig. S1C). Moreover, transfection of siRNA-resistant Lats2 restored the nuclear translocation of ASPP1 (Supplemental Fig. S1C, bottom), arguing against offtarget effects. In fact, the mere presence of a mild excess of transfected Lats2 (Supplemental Fig. S2) was sufficient to drive ASPP1 into the nucleus (Fig. 1B, middle panels). Lats2 is a serine/threonine kinase, and ASPP1 translocation was dependent on Lats2 kinase activity: Overexpression of kinase-dead Lats2 had no effect on the localization of ASPP1, which remained cytoplasmic (Fig. 1B, bottom panels).

\section{Lats2 phosphorylates ASPP1}

The inability of kinase-dead Lats2 to promote ASPP1 nuclear translocation suggested a potential role for phosphorylation of ASPP1 by Lats2. Consistent with this possibility, a predicted consensus site for Lats2 phosphorylation (Zhao et al. 2007) is present in the $\mathrm{N}$ terminus of ASPP1 (HGRSKS). Indeed, overexpression of Lats2 resulted in the appearance of a higher-molecular-weight form of ASPP1 (Fig. 2A, arrow). This form disappeared when cell lysates were treated with phosphatase (Fig. $2 \mathrm{~B})$, confirming that it was indeed due to phosphorylation. As expected, kinase-dead Lats2 failed to yield such an upshifted band (Fig. 2C). Notably, phosphorylated ASPP1 was preferentially located in the nucleus (Fig. 2D). Together, these findings strongly suggest that phosphorylation of ASPP1 by Lats 2 may drive ASPP1 into the nucleus.

\section{Lats2 and ASPP1 interact in cells}

We next determined whether Lats 2 interacted physically with ASPP1. Indeed, as shown in Figure 3B, coexpressed ASPP1 and Lats2 proteins could be coimmunoprecipitated from cell extracts, implying that they interact within cells. Using ASPP1 deletion mutants (Fig. 3A), the Lats2-binding site could be mapped to the Ankyrin (ANK) domain in the C terminus of ASPP1 (Fig. 3B): Deletion of the SH3 domain (small $\Delta \mathrm{C}$ ) did not disrupt binding, but a larger deletion of both SH3 and ANK domains $(\Delta \mathrm{C})$ no longer bound Lats2. Notably, a Lats2-induced phosphorylated form of ASPP1 was seen only with full-length ASPP1 (Fig. 3B, lane 2) but not with any of the deletion mutants, suggesting that binding of Lats2 to the ASPP1 ANK domain is required for ASPP1 phosphorylation within its N-terminal domain. Consistent with this notion, the ASPP1 $\Delta \mathrm{N}$ mutant, able to bind Lats2 (Fig. 3B, lane 5) but lacking the putative Lats2 


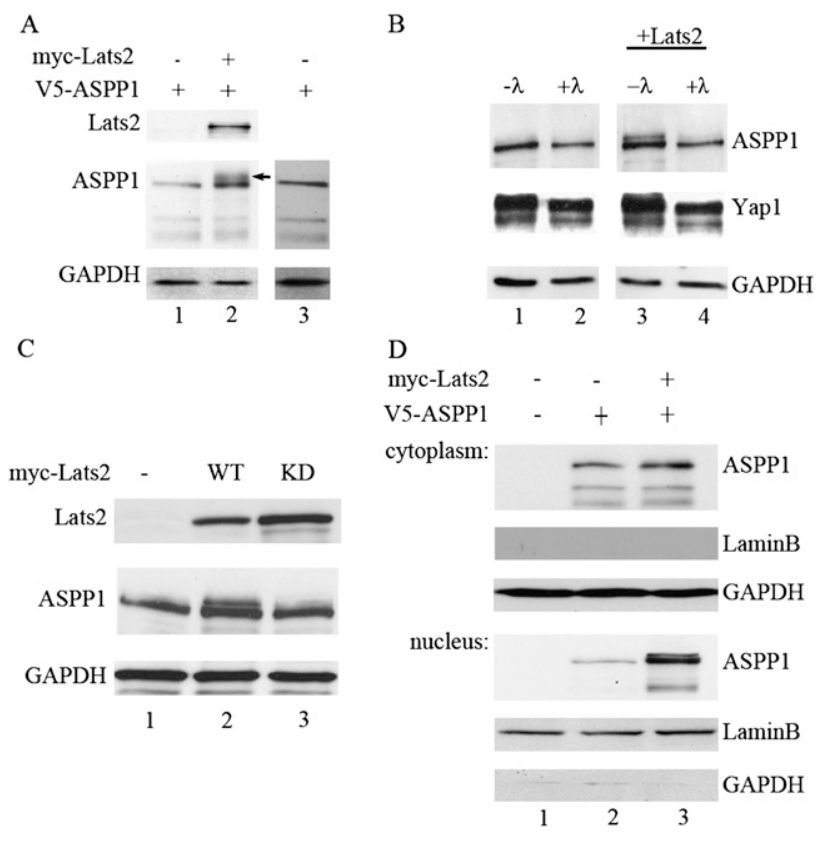

Figure 2. Lats2 phosphorylates ASPP1. (A) HCT116 cells were transiently transfected with V5-tagged ASPP1 together with empty vector or myc-tagged Lats2. Cells were harvested $24 \mathrm{~h}$ after transfection, and lysates were subjected to Western blot analysis. Lats2 and ASPP1 were detected by anti-myc tag and anti-V5 antibodies, respectively. GAPDH was used as a loading control. Lane 3 represents a longer exposure of lane 1. (B) HCT116 cells were transiently transfected as in $A$. Cell lysates were either mocktreated or treated with $\lambda$ phosphatase $(\lambda)$. Equivalent amounts were analyzed by Western blot. Ablation of phospho-Yap1 was used as a positive control for phosphatase activity. GAPDH served as a loading control. $(C)$ HCT116 cells were transiently transfected as in $A$ with empty vector, myc-tagged wild-type Lats2 (WT), or myctagged kinase-dead Lats2 (KD). GAPDH served as a loading control. (D) HCT116 cells were transiently transfected as in A. Twenty-four hours after transfection, cell lysates were processed for nuclear and cytoplasmic fractions. Equivalent amounts of each fraction were analyzed by Western blot. LaminB and GAPDH were used to verify the purity of the nuclear and cytoplasmic fractions, respectively.

phosphorylation site, was not translocated to the nucleus upon Lats2 overexpression (Supplemental Fig. S3).

Slower-migrating, presumably phosphorylated, endogenous ASPP1 also could be detected following oncogenic H-Ras expression (Fig. 3C, DW, arrow). Importantly, endogenous ASPP1 and Lats2 coimmunoprecipitated (Fig. 3C), confirming that they interact under physiological circumstances (Fig. 3C, lane 1). This interaction was significantly augmented by oncogenic Ras (Fig. 3C, cf. lanes 1 and 2). Of note, phosphorylated ASPP1 appeared to coprecipitate preferentially with Lats2 (Fig. 3C, cf. IP Lats2 and DW).

\section{Lats2 and ASPP1 mutually affect chromatin binding}

As a transcriptional regulator, ASPP1 is recruited to chromatin (Patel et al. 2008). We therefore separated chromatin-bound and chromatin-unbound cellular extract fractions. As expected, histone $\mathrm{H} 2 \mathrm{~B}$ was found primarily in the chromatin-bound fraction, while GAPDH was essen- tially chromatin-unbound (Fig. 4A). Under basal conditions, no chromatin-bound ASPP1 could be detected (Fig. 4A, lane 3). However, overexpression of oncogenic H-Ras caused a fraction of endogenous ASPP1 to associate with chromatin (Fig. 4A, lane 4), suggesting that oncogenic signals promote chromatin-related functions of ASPP1.

We next determined whether Lats2 affects the recruitment of ASPP1 to specific p53 target genes. In chromatin immunoprecipitation (ChIP) assays, ectopically expressed ASPP1 exhibited a modest association with $\mathrm{p} 53$ response genes (Fig. 4B), compared with control HA antibody (Supplemental Fig. S4A), but not with GAPDH, serving as a negative control (Supplemental Fig. S4B). Coexpression of Lats2 significantly enhanced the binding of ASPP1 to several (CD95, BAX, and Gaddd45a), albeit not all (PUMA), apoptosis-related genes (Fig. 4B). Interestingly, this was not the case for cell cycle regulatory genes such as p21 and Btg2, suggesting that the effect of Lats2 on ASPP1 chromatin association is gene-specific, and that Lats2 may modulate the biological outcome of nuclear ASPP1 activity.

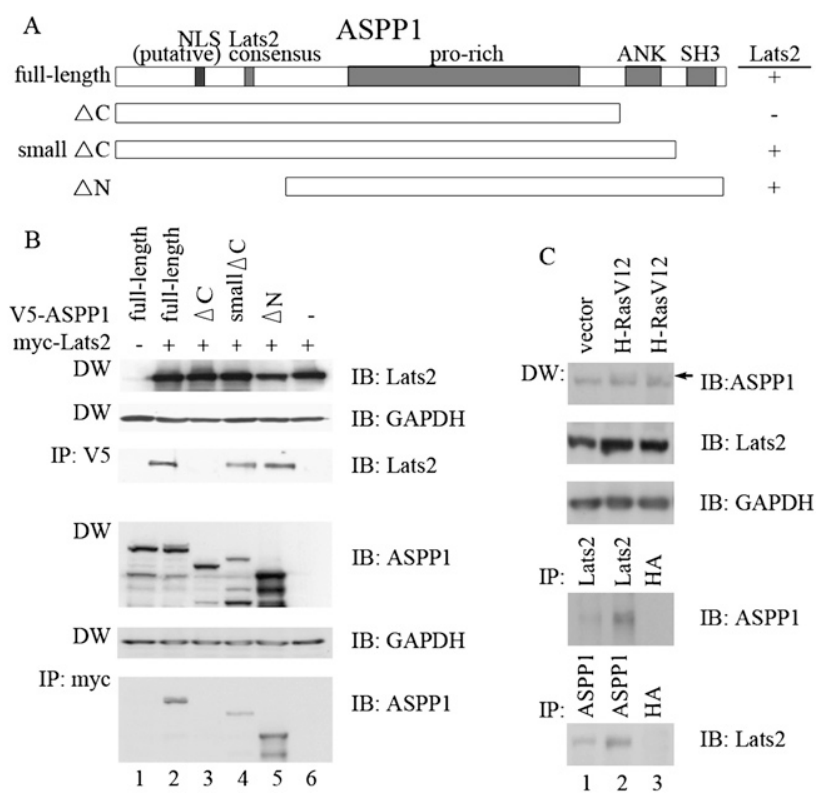

Figure 3. Lats 2 and ASPP1 interact within cells. (A) Schematic representation of full-length ASPP1 and different deletion mutants lacking either the $\mathrm{N}$ terminus $(\Delta \mathrm{N})$, the ANK repeat (ANK), and SH3 domain $(\Delta \mathrm{C})$, or the SH3 domain only (small $\Delta C)$. (B) HCT116 cells were transiently transfected with V5-tagged full-length ASPP1 or deletion mutants thereof, together with empty vector or myc-tagged Lats2. Lysates were immunoprecipitated (IP) with either anti-V5 or anti-myc antibodies. Westerns were immunoblotted (IB) using antibodies directed against either V5 tag, myc tag, or GAPDH. (DW) Direct Western: 2.5\% of each lysate subjected directly to SDS-PAGE and Western blot analysis. (C) WI-38 cells were infected with H-RasV12 or empty retroviral vector. Two days after infection, cells were harvested and subjected to immunoprecipitation with either anti-Lats2, anti-ASPP1, or control anti-HA antibodies. Membranes were immunoblotted (IB) using anti-ASPP1, anti-Lats2 (LA2), or anti-GAPDH antibodies. Direct Western (DW) is as in $B$. 
A

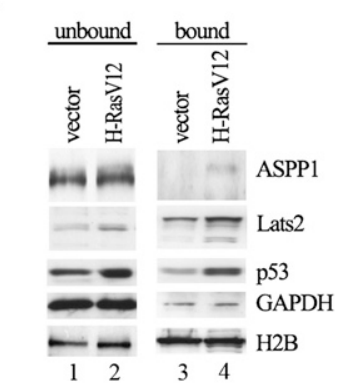

C
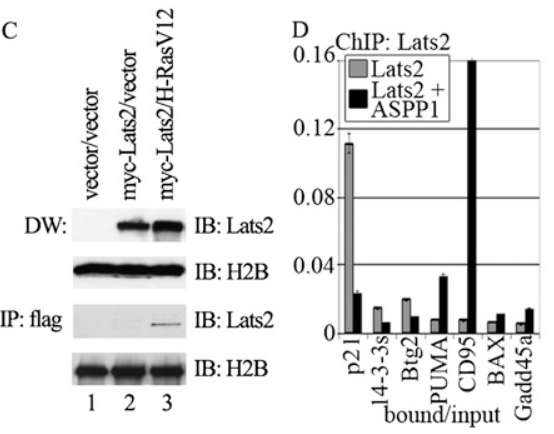
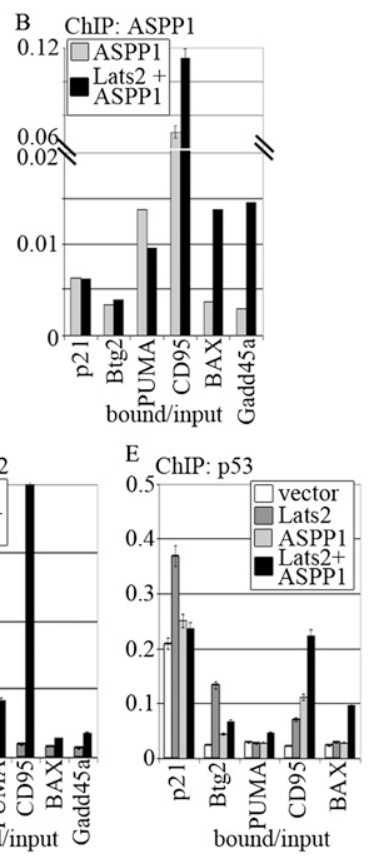

Figure 4. Lats2 and ASPP1 shift p53 binding toward proapoptotic promoters. $(A)$ HCT116 cells were transiently transfected with vector or H-RasV12. Twenty-four hours after transfection, cell lysates were separated into chromatin-bound and chromatinunbound fractions. Equivalent amounts of each fraction were analyzed by Western blot using antibodies against ASPP1, Lats2, and p53. H2B and GAPDH were used to verify the purity of the bound and unbound fractions, respectively. (B) HCT116 cells were transiently transfected with the indicated combinations of V5-tagged ASPP1 and myc-tagged Lats2. Forty-eight hours later, ChIP was performed using antibodies against V5 tag to immunoprecipitate ASPP1. Input and bound DNA were quantified using qPCR with primer pairs corresponding to p53-responsive elements within the indicated genes. (C) HCT116 cells were transiently transfected with Flag-tagged H2B together with the indicated combinations of vector, myc-tagged Lats2, and H-RasV12. Lysates were immunoprecipitated (IP) using anti-Flag antibodies and then eluted using Flag peptide. Westerns were immunoblotted (IB) using anti-myc or anti-Flag antibodies to detect Lats2 or $\mathrm{H} 2 \mathrm{~B}$, respectively. Direct Western (DW) is as in Figure 3B. $(D, E)$ ChIP was performed as in $B$, except that anti-myc tag or anti-p53 (CM1) antibodies were used to precipitate Lats2 or p53, respectively.

Both Lats2 and p53 accumulated in response to expression of $\mathrm{H}-\mathrm{RasV12}$, and this resulted in a proportionate increase in the chromatin-bound subpopulation of both proteins (Fig. 4A). Moreover, Lats2 could be coimmunoprecipitated with histone $\mathrm{H} 2 \mathrm{~B}$ in cells expressing oncogenic H-Ras (Fig. 4C, lane 3), further demonstrating that it becomes preferentially associated with chromatin under such conditions. Hence, oncogenic H-RasV12 not only promotes Lats2-dependent phosphorylation and nuclear import of ASPP1, but also mobilizes Lats2 itself to play a chromatin-associated role in the nucleus, presumably affecting the transcription of specific genes.

To further explore the chromatin-associated functions of Lats2, we performed ChIP experiments to examine the association of Lats2 with different p53 target genes. Lats2

was detected strongly on the p 21 promoter, but only minimally on the p53 response elements associated with a number of other genes (Fig. 4D). Surprisingly, coexpression of ASPP1 dramatically diminished the association of Lats2 with the p21 promoter ( $\sim 5.5$-fold). At the same time, Lats2 became increasingly associated with other genes, such as PUMA, CD95, BAX, and Gadd45a (Fig. 4D). Thus, Lats2 and ASPP1 mutually augment each other's recruitment to a subset of p53 target genes, each of which can contribute to apoptosis (Espanel and Sudol 2001; Labi et al. 2006; Tront et al. 2006; Chiou and Hoa 2009; Schilling et al. 2009). Interestingly, shRNA-mediated knockdown of endogenous p53, albeit partial (Supplemental Fig. S4C), markedly abrogated Lats2 binding to the CD95 promoter (Supplemental Fig. S4D). In contrast, binding of Lats2 to the osteocalcin promoter was only mildly affected by p53 knockdown. Hence, p53 is required for the recruitment of Lats2 to p53 target genes. Of note, kinase-dead Lats2 failed to associate with the CD95 promoter (Supplemental Fig. S4D, Lats2 kd), implying that Lats2 kinase activity may be important in facilitating its chromatin-associated functions.

Sequence-specific DNA binding of endogenous p53 was also affected by Lats 2 and ASPP1. For instance, $\mathrm{p} 53$ binding to the cell cycle-related p21 and Btg2 genes was significantly enhanced by Lats2 overexpression, but less so by ASPP1 (Fig. 4E). In contrast, binding of p53 to the PUMA, CD95, and BAX genes was only modestly induced, if at all, by Lats2 alone or ASPP1 alone, but was markedly enhanced upon coexpression of Lats2 and ASPP1 together (Fig. 4E). ASPP1 has been reported to increase the binding of p53 to its proapoptotic target genes (Samuels-Lev et al. 2001); our data imply that Lats2 is required in order to enable ASPP1 to do so efficiently. Together, the findings described above suggest that nuclear Lats2 and nuclear phospho-ASPP1 cooperate to shift p53 binding toward proapoptotic target genes, presumably through formation of a p53-Lats2ASPP1 complex.

\section{Lats2 and ASPP1 modulate p53 target gene transcription}

Consistent with their effects on p53 DNA binding, expression of ASPP1 and Lats2 also affected p53 transcriptional output. Thus, in luciferase reporter assays, exogenous Lats2 augmented markedly the transactivation of the p21 and Cyclin G1, but not the Pig3 or Bax, promoters (Fig. 5A; data not shown). Substantial activation of the Pig3 and Bax promoters required the overexpression of both Lats2 and ASPP1 together or either one in combination with 5-fluorouracil (5FU), a p53-activating anti-cancer drug (Fig. 5A). Moreover, the effects of Lats2 and ASPP1 were p53-dependent, since, in isogenic cells lacking p53, their effect was dramatically reduced (Supplemental Fig. S5A). Similarly, in H-RasV12-expressing cells, transcription of endogenous proapoptotic genes was dependent on both Lats2 and ASPP1. Knockdown of either Lats2 or ASPP1 significantly diminished the induction of CD95, Pig3, and Bax mRNA by oncogenic stress (Fig. 5B; Supplemental Figs. S1B [knockdown validation], S5B). 

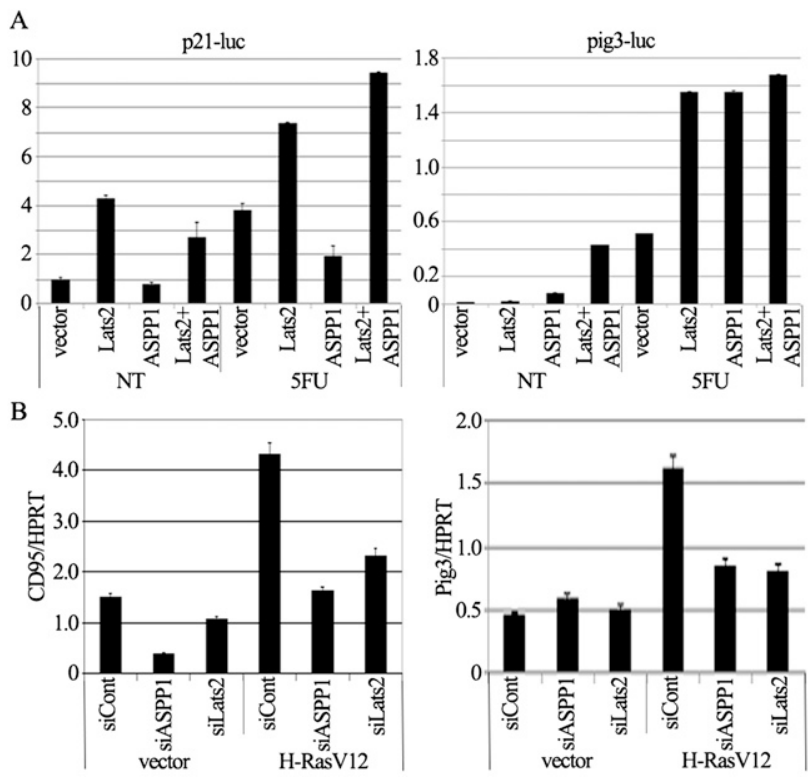

Figure 5. Lats2 and ASPP1 modulate p53 target gene preference. (A) HCT116 cells were transiently transfected with combinations of vector, ASPP1, and Lats2 together with the indicated firefly luciferase reporter plasmids. Twenty-four hours later, cells were either mock-treated or treated with 5-FU (50 $\mu \mathrm{g} / \mathrm{mL}$ ) for $16 \mathrm{~h}$. Luciferase assays were performed in triplicate, and values represent average firefly luciferase expression after normalization for cotransfected renilla luciferase plasmid. $(B)$ WI-38 cells were infected with H-RasV12 or empty retroviral vector. Two days later, cells were transiently transfected with siRNA oligonucleotides specific for ASPP1 or Lats2 or control siRNA (siASPP1, siLats2, and siCont, respectively). Three days after infection, cells were harvested, and RNA was extracted and subjected to qRT-PCR analysis. Values were normalized to HPRT mRNA.

\section{Lats2 and ASPP1 mediate apoptosis of polyploid cells}

Within $4 \mathrm{~d}$ of expression of oncogenic H-Ras in WI-38 cells, some of the cells undergo apoptosis (Fig. 6A,B; Aylon et al. 2009). This apoptosis is almost entirely dependent on both Lats2 and ASPP1, since knockdown of either gene markedly reduced apoptosis and brought it back to basal levels. Concomitant with the diminished apoptosis, a similar fraction of the cells depleted of Lats2 or ASPP1 emerged with polyploid genomes (>4N DNA content) (Fig. 6A,B; Supplemental Fig. S6A). Notably, a large proportion of the polyploid cells stained positive for activated caspase 3 (Supplemental Fig. S6B), directly demonstrating that they were selectively undergoing apoptosis. Furthermore, treatment with the pan-caspase inhibitor Z-VAD-FMK, which completely abrogated caspase activation (Supplemental Fig. S6B), markedly augmented the $>4 \mathrm{~N}$ fraction at the expense of the apoptotic sub-G1 fraction (Supplemental Fig. S6C), further confirming that polyploid cells were subject to apoptotic elimination in this system.

Together, these results support a model wherein oncogenic stress causes Lats2-dependent phosphorylation of ASPP1. Phosphorylated ASPP1 accumulates in the nucleus and binds preferentially to chromatin. Moreover, Lats2 and ASPP1 cooperate to instigate a proapoptotic p53 transcriptional program that preferentially kills polyploid cells.

\section{Yap1 antagonizes Lats2-ASPP1 function}

The function of Yap1 in tumorigenesis remains ambiguous, with seemingly conflicting reports of its roles as a tumor suppressor (Lapi et al. 2008) or oncogene (Overholtzer et al. 2006). Previously, Yap1 was identified as a phosphorylation target of Lats2 (Kawahara et al. 2008). Interestingly, Yap1 already has been shown to bind ASPP2 (Espanel and Sudol 2001). For these reasons, and given the similarity between ASPP1 and ASPP2, we sought to examine the effect of Yap1 in our system.

GFP-tagged Lats2 localized to centrosomes; following overexpression of H-RasV12, it translocated to and accumulated in the nucleus (Fig. 6C, top and middle panels) as

A
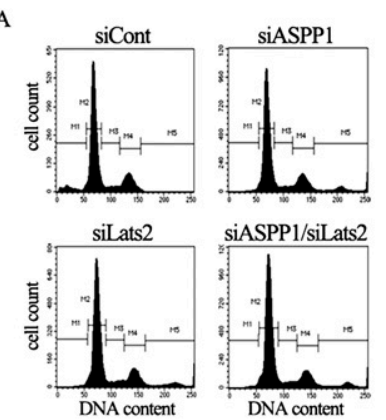
siASPP1/siLats2
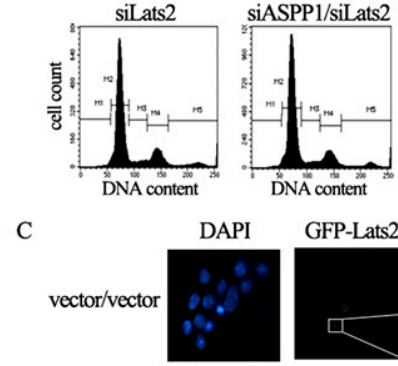

GFP-Lats2
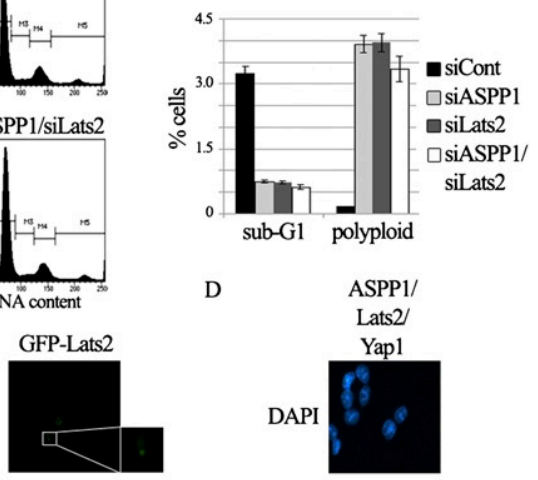

H-RasV12/vector
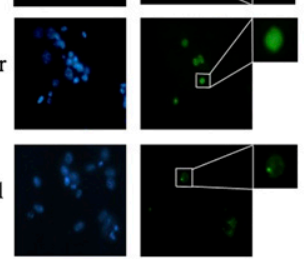

D

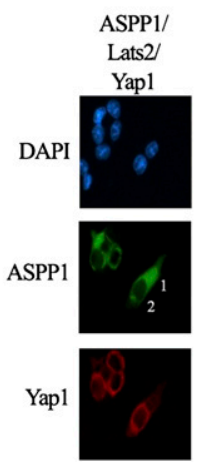

Figure 6. Lats2 and ASPP1 promote apoptosis of polyploid cells. (A) WI-38 cells were infected with H-RasV12 or empty retroviral vector. Two days after infection, cells were transfected with siRNA oligonucleotides specific for ASPP1 or Lats2 or control siRNA (siASPP1, siLats2, and siCont, respectively). Three days after infection, cells were harvested, fixed, stained with PI, and subjected to FACS-based DNA content analysis. (B) Graphic representation of averaged values corresponding to the indicated cell subpopulations in $A$. $(C)$ HCT116 cells were transiently transfected with GFP-tagged Lats2, together with the indicated combinations of H-RasV12 and Flag-tagged Yap1. Twenty-four hours later, cells were fixed and subjected to analysis by light microscopy. Nuclear DNA was detected by DAPI staining. Single representative cells are magnified and presented in the far right panel. (D) HCT116 cells were transiently transfected with V5-tagged ASPP1, myc-tagged Lats2, and Flag-tagged Yap1. Twenty-four hours later, cells were fixed and immunostained using anti-V5 or anti-Flag antibodies. Nuclear DNA was detected by DAPI staining. Cells marked 1 and 2 are representative examples of nuclear and cytoplasmic ASPP1 staining, respectively. 
expected (Aylon et al. 2009). Surprisingly, overexpression of Yap1 diminished the ability of Lats2 to depart from centrosomes and accumulate in the nucleus in response to H-RasV12 (Fig. 6C, bottom panels). Thus, in addition to being a phosphorylation target of Lats2, Yap1 can also act upstream of Lats2 and inhibit its nuclear function. Overexpression of Yap1 also thwarted the nuclear accumulation of ASPP1 in response to Lats2 overexpression (cf. Figs. 6D and 1B). In fact, the ability of Lats2-overexpressing cells to accumulate nuclear ASPP1 appeared to be inversely correlated to Yap1 protein levels (Fig. 6D, cf. cells labeled 1 and 2, for instance).

Overexpression of Yap1 diminished, in a dose-dependent manner, the Lats2-dependent upshift of ASPP1 (Fig. 7A, second panel, lanes 4-6), suggesting that Yap1 has an inhibitory effect on the kinase activity of Lats2 toward ASPP1. Indeed, increasing amounts of Yap1 disrupted the coprecipitation of Lats2 with ASPP1 (Fig. 7A, second panel from bottom; Vigneron et al. 2010), providing a mechanistic explanation for the inhibition of ASPP1 phosphoryla-

A

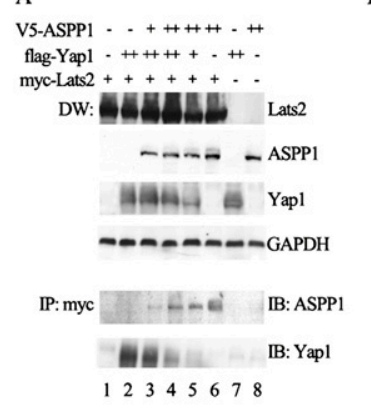

B

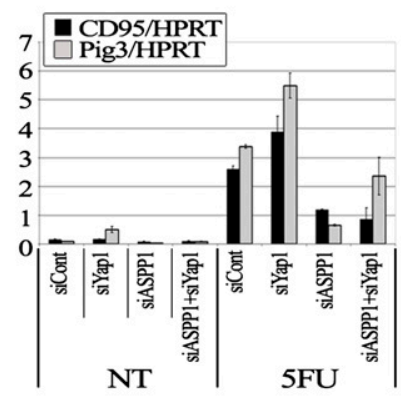

C

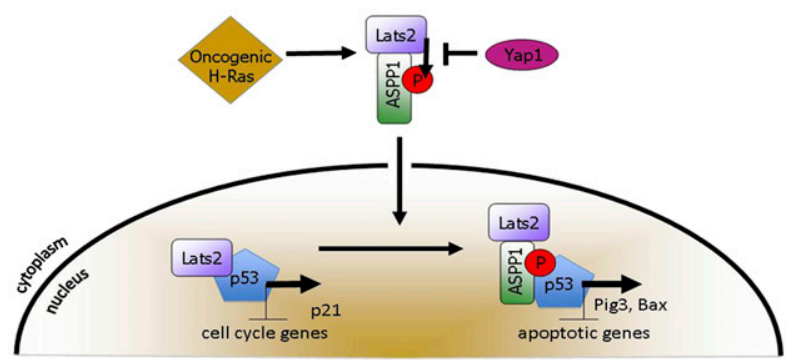

Figure 7. Yap1 antagonizes the effects of Lats2 and ASPP1. (A) HCT116 cells were transiently transfected with combinations of V5-tagged ASPP1 $(0.5 \mu \mathrm{g}[+]$ or $2 \mu \mathrm{g}[++])$, Flag-tagged Yap1 $(0.5$ $\mu \mathrm{g}[+]$ or $2 \mu \mathrm{g}[++])$, and myc-tagged Lats $2(3 \mu \mathrm{g}[+])$. Anti-myc tag antibodies were used to coimmunoprecipitate Lats2. Western blot analysis was performed; Lats2, ASPP1, and Yap1 were visualized using anti-myc tag, anti-V5 tag, and anti-Flag antibodies, respectively. Direct Western (DW) is as in Figure 3B. GAPDH was used as a loading control. $(B)$ HCT116 cells were transiently transfected with combinations of siRNA oligonucleotides specific for Yap1 and ASPP1, or control siRNA (siYap1, siASPP1, and siCont, respectively). Twenty-four hours later, cells were either mocktreated or treated with 5 -FU $(50 \mu \mathrm{g} / \mathrm{mL})$ for $16 \mathrm{~h}$. Cells were then harvested, and RNA was extracted and subjected to qRT-PCR analysis. Values were normalized to HPRT mRNA. (C) Proposed model for cross-talk between oncogenic Ras, Lats2, APP1, Yap1, and $\mathrm{p} 53$. tion. Moreover, excess Yap1 abrogated the coprecipitation of phosphorylated ASPP1 with Lats2. Conversely, Yap1 and Lats2 robustly coprecipitated in the absence of ASPP1 (Fig. 7A, bottom panel, lane 2), but this interaction was abrogated by increasing amounts of ASPP1 (Fig. 7A, bottom panel, lanes 3,4), implying that Yap1 and ASPP1 interfere with the binding of each other to Lats2.

This possibility was further explored using Lats2 deletion mutants (Supplemental Fig. S7A). A polypeptide comprising residues 255-621 of Lats2 strongly bound Yap1 (Supplemental Fig. S7B, top panel), but was not sufficient for ASPP1 binding. On the other hand, deletion of this region $(\Delta \mathrm{P})$ abrogated both Yap1 and ASPP1 binding. This suggests that binding of ASPP1 might require multiple sites on Lats2 through either formation of multiple protein-protein contacts or conformational effects. The observed functional competition (Fig. 7A) thus might be due to either mutual steric interference or conformational changes induced by either interaction.

Consistent with its recruitment to p53-binding sites on chromatin, Lats2 could be coprecipitated with p53 (Supplemental Fig. S7C, lanes 2-4). Notably, this interaction was almost completely abolished by Yap1 overexpression (Supplemental Fig. S7C, lane 5), presumably owing to exclusion of Lats2 from the nucleus (see Fig. 6C). Accordingly, Yap1 attenuated the Lats2-mediated transcriptional output of p53. Thus, in luciferase reporter assays, Yap1 overexpression greatly compromised Lats2-driven transcriptional activation of the p21 and Pig3 promoters (Supplemental Fig. S8A). Conversely, down-regulation of endogenous Yap1 (Fig. 7B; Supplemental Fig. S8B, knockdown validation) caused increased expression of ASPP1dependent transcripts under both basal (NT and Pig3) and stress-induced (5-FU, Pig3, and CD95) conditions.

Notably, Yap1 overexpression strongly suppressed the induction of apoptosis by p53 in conjunction with Lats2 and ASPP1, concomitant with an increase in polyploid cells (Supplemental Fig. S8C). Conversely, excess Lats2 and ASPP1 could override the ability of Yap1 to induce increased migration of U2OS osteosarcoma cells (Supplemental Fig. S8D). Together, these data suggest that some of the cancer-promoting effects of Yap1 may be partially attributed to inactivation of the Lats2-ASPP1 pathway.

In conclusion, we show that the Lats2-ASPP1-p53 axis functions in a distinctive tumor suppression pathway. However, high levels of Yap1 can antagonize this function, hampering the p53 transcriptional program in a manner that might then permit tumorigenesis to proceed.

\section{Discussion}

We reported previously that Lats2 binds to $\mathrm{Mdm} 2$ and inhibits its ability to promote p53 ubiquitination and degradation (Aylon et al. 2006). As now shown, Lats2 also drives the phosphorylation and nuclear translocation of ASPP1, a proapoptotic $\mathrm{p} 53$ transcriptional cofactor. Moreover, Lats2 interacts with p53 and is recruited to p53responsive genes in a p53-dependent manner. Lats2 can also act downstream from $\mathrm{p} 53$ and $\mathrm{p} 21$ to suppress cyclindependent kinase activity (Voorhoeve et al. 2006). Hence, 
Lats2 interfaces with the p53 pathway at multiple levels, potentially rendering it a pivotal regulator of p53 function. The involvement of Lats2 appears to be particularly meaningful under conditions of oncogenic stress induced by mutant Ras (Voorhoeve et al. 2006; Aylon et al. 2009). It is thus not surprising that Lats2 promoter hypermethylation strongly favors Ras-initiated transformation of p53-proficient cells (Aylon et al. 2009).

Phosphorylated ASPP1 accumulates in the nucleus and drives p53-dependent apoptosis. High levels of Yap1 override this Lats2-ASPP1-p53 transcriptional program in a manner that might facilitate tumorigenesis (Fig. 7C). Conversely, when retained in the cytoplasm, ASPP1 may exert anti-apoptotic and procancer effects by augmenting Yap1 stability and nuclear localization, as described by Vigneron et al. (2010).

In combination, our data suggest that the relative levels of Lats2, ASPP1, and Yap1 proteins might be important for determining whether p53 can instigate programmed cell death in neoplastic cells. In support of this idea, decreased Lats2 expression has been linked to clinically aggressive breast cancer (Takahashi et al. 2005), astrocytoma (Jiang et al. 2006), acute lymphoblastic leukemia (Jimenez-Velasco et al. 2005), and non-small-cell lung carcinoma (Strazisar et al. 2009; Liu et al. 2010) tumors. Likewise, ASPP1 expression is down-regulated in breast cancer, hepatocellular carcinoma (Samuels-Lev et al. 2001; Liu et al. 2005), and leukemia (J Zhao et al. 2010), where it is associated with a high relapse rate and poor prognosis (Agirre et al. 2006). Furthermore, in line with our results, ASPP1 has been shown to reduce the transforming activity of Ras (Bergamaschi et al. 2003), whereas elevated expression of Yap1 has been associated with numerous cancers (Dong et al. 2007; Zhao et al. 2007; Steinhardt et al. 2008; Fernandez et al. 2009). Our working model predicts that altered expression of any single component (Lats2, ASPP1, Yap1, or p53 itself) might confer a sufficient selective advantage to allow tumorigenesis to proceed with no need for additional alterations in this pathway.

Intriguingly, all three proteins (Lats2, Yap1, and ASPP1) can reside in either the cytoplasm or the nucleus, presumably serving different purposes in the different locations. We showed previously that Lats2 could translocate from the centrosome to the nucleus in response to mitotic or oncogenic stress (Aylon et al. 2006, 2009). Yap1 translocation from the cytoplasm to the nucleus is dependent on ASPP1 levels (Vigneron t al. 2010) and cell density (Oka and Sudol 2009; B Zhao et al. 2010). In the present study, we show that ASPP1, too, is subject to regulated cytoplasmic-nuclear translocation. Interestingly, the ASPP1 protein sequence encodes a putative nuclear export sequence (NES) (depicted schematically in Fig 3A) adjacent to the Lats2 phosphorylation site, raising the possibility that phosphorylation by Lats 2 may cause ASPP1 nuclear accumulation by masking this NES. Our findings may thus provide a missing link to explain the apparent discrepancy between the reported cytoplasmic location of ASPP1 and its transcriptional effects in the nucleus (Sullivan and Lu 2007).

We propose that, in normal cells, a delicate balance between the levels of Lats2, ASPP1, and Yap1 and the tight control of their location is key to regulating cell fate. Lats2-mediated Yap1 destabilization (B Zhao et al. 2010) and increased Yapl cytoplasmic sequestration, primarily via phosphorylation of Yap1 Ser127, act to restrict Yap1 oncogenic functions (Zhao et al. 2007). Additionally, phosphorylation and elimination of Yap1 might facilitate the association of Lats2 with ASPP1 and enhance their tumorsuppressing function. In this scenario, phosphorylation by Lats 2 has a dual effect, acting on both partners to prevent their undesirable oncogenic functions. In cancers with diminished Lats2 activity, Yap1 would remain nuclear and ASPP1 would remain cytoplasmic; both of these features have been associated with anti-apoptotic, progrowth functions (Oka and Sudol 2009; Vigneron et al. 2010).

Interestingly, in some cases, YAP, in association with the tumor suppressor p73, acts to promote apoptosis /Oka and Sudol 2009). Hence, YAP may impinge on two pivotal tumor suppressor pathways, either directly via p73 (Strano et al. 2001) or indirectly by modulating Lats2-ASPP1-p53 function (this study).

Where, within the cell, are the binding of Lats2 to ASPP1 and the phosphorylation of ASPP1 by Lats2 taking place? Under some conditions, ASPP1 can be located at the centrosome (Thornton et al. 2006), where Lats2 normally resides. However, although knockdown of Lats2 abolishes both the phosphorylation of ASPP1 and its nuclear import upon oncogenic stress, this does not cause ASPP1 to accumulate at the centrosome (Fig. 1A). This is in line with our previous observations that oncogenic $\mathrm{H}$-Ras triggers the departure of Lats2 from centrosomes and a subsequent ATR-Chk1-dependent translocation of Lats2 from the cytoplasm into the nucleus (Aylon et al. 2009). We therefore speculate that ASPP1 might be phosphorylated in the cytoplasm by oncogene-activated Lats2.

To date, not much is known about the transcriptional function of Lats2. Lats2 was reported previously to act as a transcriptional repressor interfering with oncogenic androgen receptor target induction (Powzaniuk et al. 2004). Here, we report that Lats2 facilitates gene transactivation by the p53 tumor suppressor. We also detected Lats2 on the osteocalcin promoter, which is a target of Runx transcription factors but not of p53 (Zaidi et al. 2004). Lats2 may thus play an as yet underappreciated role as a transcriptional coregulator.

In conclusion, the present study delineates a new axis composed of Lats2, ASPP1, and p53 whose tumor suppressor activity is dependent on the presence of its individual components within the nucleus. Conditions that interfere with the nuclear translocation of ASPP1 and Lats2, as exemplified here by Yap1 overexpression, will therefore disrupt the functionality of this axis and instead promote tumor progression. Furthermore, as illustrated by Vigneron et al. (2010), cytoplasmic retention of ASPP1 will convert it from a proapoptotic effector into an oncogenic protein, contributing to augmented malignant cell properties. Together with the data reported in the accompanying study by Vigneron et al. (2010), our findings delineate an intricate set of cross-regulatory interactions between Yap1, Lats2, and ASPP1. The balance between these interactions can dictate whether a cell will acquire 
cancerous features, or whether tumor suppression will prevail.

\section{Materials and methods}

\section{Plasmids}

The following plasmids were used: pBabe-puro and pBabeH-RasV12-puro; pCDNA3, pCDNA3-myc-Lats2, pCDNA3-mycLats2-KD, and pCDNA3-GFP-Lats2 (Toji et al. 2004); pCDN3V5-ASPP1 (and derivatives) (Samuels-Lev et al. 2001); and pCDNA3-flag-Yap1 (generous gift from Y. Shaul).

\section{Cell culture, transfections, and viral infections}

All cells were maintained at $37^{\circ} \mathrm{C}$. hTert immortalized WI-38 "fast-growers" expressing ecotropic receptor (referred to in the text as WI-38 cells) (Milyavsky et al. 2003) were grown in MEM supplemented with $10 \%$ non-heat-inactivated fetal bovine serum (FBS) (Sigma), MEM-Eagle nonessential amino acids, sodium pyruvate, L-glutamine (Beit HaEmek), and antibiotics. HCT116 p53 $3^{+/+}$and HCT116 p53 $3^{-/-}$(generous gift of B. Vogelstein) cells were grown in McCoy's medium supplemented with $10 \%$ heatinactivated FBS (HI-FBS, Sigma), 2 mM L-glutamine, and antibiotics. HCT116 cells were transfected using JetPEI reagent (PolyPlus Transfection). WI-38 cells were virally infected and selected in the presence of the appropriate antibiotic drug. For siRNAmediated knockdown, $2 \mathrm{~d}$ after infection, cells were transfected with appropriate SMARTpools (Dharmacon) and transfection reagents according to the manufacturer's instructions.

\section{Western blots analysis}

Western blot analysis was performed as described (Aylon et al. 2006). Antibodies directed against the following antibodies were used: Lats2 (LA-2) (Toji et al. 2004), GAPDH (Chemicon), p53 (mixture of DO1 + PAb1801), ASPP1 (Novus), H2B (Upstate Biotechnologies), Yap1 (Santa Cruz Biotechnology), LaminB (Santa Cruz Biotechnology), V5-tag (Invitrogen), myc-tag (9E10), Flag tag (Sigma), and Mdm2 (mixture of 4B2, 2A9, and 4B11).

\section{Isolation of total RNA and real-time quantitative} PCR ( $q P C R)$

Total RNA was isolated using NucleoSpin RNA II kit (MachereyNagel). A 1.5- $\mu$ g aliquot of the total RNA was reverse-transcribed using Moloney murine leukemia virus reverse transcriptase enzyme (Promega) and random hexamer primers (Amersham). Real-time qPCR was performed using SYBR Green Master Mix (Applied Biosystems) on an ABI 7300 instrument (Applied Biosystems). The values for specific genes were normalized to hypoxanthine phosphorlbosyltransferase (HPRT). Primer sequences are detailed in the Supplemental Material.

\section{Immunofluorescence}

Cells were plated and grown on 12-mm coverslips and fixed with ice-cold methanol for $20 \mathrm{~min}$ at $4^{\circ} \mathrm{C}$. After gradual rehydration with PBS, cells were washed with PBS and then blocked with $3 \%$ BSA in PBS. Samples were then incubated for $1 \mathrm{~h}$ with primary antibody, washed, and then incubated with secondary antibody and DAPI ( $5 \mathrm{mg} / \mathrm{mL}$ final) for $40 \mathrm{~min}$ in the dark. To detect Lats2, anti-KPM/Lats2 (Santa Cruz Biotechnology) or LA-2 antibodies were used. Other antibodies used for staining included antiASPP1 (Sigma) and anti-Flag (Sigma). DAPI (Sigma) was used to visualize nuclear DNA.

\section{Cell cycle and BrdU analyses}

Cells were prelabeled with $10 \mu \mathrm{M}$ BrdU (Sigma). After $45 \mathrm{~min}$, cells were detached with trypsin, collected, and fixed with $70 \%$ $\mathrm{EtOH} / \mathrm{HBSS}$ overnight at $-20^{\circ} \mathrm{C}$. Fixed cells were denatured $(2 \mathrm{~N}$ $\mathrm{HCl}, 0.5 \%$ Triton in PBS) for $30 \mathrm{~min}$ at room temperature and then neutralized with $0.1 \mathrm{M} \mathrm{Na}_{2} \mathrm{~B}_{4} \mathrm{O}_{7}(\mathrm{pH} 8.5)$, incubated with anti-BrdU antibodies (Becton Dickinson) for $1 \mathrm{~h}$, and resuspended in $50 \mu \mathrm{g} / \mathrm{mL}$ propidium iodide.

\section{Cell fractionation}

Trypsinized cells were resuspended in RSB buffer $(10 \mathrm{mM} \mathrm{NaCl}$, $10 \mathrm{mM}$ Tris at $\mathrm{pH} 7.4,15 \mathrm{mM} \mathrm{MgCl}_{2}$ ) including protease inhibitor mix (Sigma), and then lysed with 10\% Triton and 10\% deoxycholate. Nuclei were separated from cytoplasm by centrifugation at $14,000 \mathrm{rpm}$ for $10 \mathrm{~min}$.

\section{Phosphatase treatment}

Trypsinized cells were resuspended in $\lambda$ phosphatase buffer $(1 \%$ Triton X-100, 0.1\% SDS, 0.5\% NP-40, $150 \mathrm{mM} \mathrm{NaCl}, 30 \mathrm{mM}$ Tris at $\mathrm{pH} 7.4,2.5 \mathrm{mM}$ EDTA supplemented with protease inhibitor mix [Sigma]). After centrifugation, the supernatant was made $7.5 \mathrm{mM} \mathrm{MgCl}_{2}$ and $7.5 \mathrm{mM} \mathrm{MnCl}_{2}$ : Half of the lysate was treated with $800 \mathrm{U}$ of $\lambda$ phosphatase for $1 \mathrm{~h}$ at $30^{\circ} \mathrm{C}$, while the other half was mock-treated.

\section{Coimmunoprecipitation analysis}

The monolayer was gently washed twice with ice-cold PBS and lysed for $30 \mathrm{~min}$ on ice with NP-40 lysis buffer $(50 \mathrm{mM}$ Tris. $\mathrm{HCl}$ at $\mathrm{pH} 8.0,150 \mathrm{mM} \mathrm{NaCl}, 1.0 \% \mathrm{NP}-40)$ supplemented with protease inhibitor mix (Sigma) and phosphatase inhibitor cocktail I + II (Sigma). Protein A-Sepharose beads (Repligen), preblocked with BSA, were incubated with appropriate antibodies. Lysates were incubated with the bound antibodies for $4 \mathrm{~h}$ at $4^{\circ} \mathrm{C}$, washed with NP-40 lysis buffer, released from beads by boiling, and resolved by SDS-PAGE.

\section{Chromatin-bound and chromatin-unbound fractions}

The monolayer was gently washed twice with ice-cold PBS and incubated for $5 \mathrm{~min}$ on ice with $100 \mathrm{mM} \mathrm{NaCl}, 300 \mathrm{mM}$ sucrose, $3 \mathrm{mM} \mathrm{MgCl}_{2}, 10 \mathrm{mM}$ Pipes (pH 6.8), 1 mM EGTD, 0.2\% Triton $\mathrm{X}-100$, and protease inhibitor mix (Sigma) to isolate the unbound fraction. The remaining bound fraction was washed with PBS and then harvested in $100 \mathrm{mM} \mathrm{NaCl}, 300 \mathrm{mM}$ sucrose, $3 \mathrm{mM}$ $\mathrm{MgCl}_{2}, 10 \mathrm{mM}$ PIPES (pH 6.8), $1 \mathrm{mM}$ EGTA, and $0.5 \mathrm{U} / \mu \mathrm{L}$ DNaseI with protease inhibitor mix (Sigma).

\section{ChIP}

ChIP analysis was performed as described (Minsky and Oren 2004). Precipitated DNA was subjected to qPCR analysis using gene-specific primer pairs. Primer sequences are detailed in the Supplemental Material.

\section{Luciferase assays}

Cells were plated in 12-well dishes at $5 \times 10^{4}$ cells per well, and were transfected $24 \mathrm{~h}$ later with the indicated firefly luciferase reporter plasmids. All transfections included also a constant amount of Renilla luciferase plasmid for internal control. Twenty-hours later, cells were incubated for $30 \mathrm{~min}$ with 100 $\mu \mathrm{L}$ of passive lysis buffer (Amersham) per well, and $35 \mu \mathrm{L}$ of each 
lysate was subjected to a dual-luciferase assay (Promega) using a Luminoskan Ascent apparatus (Thermo LabSystems). Results of triplicate transfections are presented after normalization to Renilla luciferase activity in the corresponding samples \pm SEM.

\section{Acknowledgments}

We thank Michal Natan and Steven Woods for helpful experimental contributions, Moshe Yaniv for critical reading of the manuscript, Sylvia Wilder for excellent technical assistance, and Efrat Lidor-Nili for ChIP advice. This work was supported in part by grant R37 CA40099 from the National Cancer Institute, the Dr. Miriam and Sheldon Adelson Medical Research Foundation, the M.D. Moross Institute for Cancer Research, and the Yad Abraham Center for Cancer Diagnosis and Therapy. M.O. is incumbent of the Andre Lwoff chair in Molecular Biology.

\section{References}

Agirre X, Roman-Gomez J, Jimenez-Velasco A, Garate L, MontielDuarte C, Navarro G, Vazquez I, Zalacain M, Calasanz MJ, Heiniger A, et al. 2006. ASPP1, a common activator of TP53, is inactivated by aberrant methylation of its promoter in acute lymphoblastic leukemia. Oncogene 25: 1862-1870.

Aylon Y, Michael D, Shmueli A, Yabuta N, Nojima H, Oren M. 2006. A positive feedback loop between the p53 and Lats2 tumor suppressors prevents tetraploidization. Genes Dev 20: 2687-2700.

Aylon Y, Yabuta N, Besserglick H, Buganim Y, Rotter V, Nojima H, Oren M. 2009. Silencing of the Lats2 tumor suppressor overrides a p53-dependent oncogenic stress checkpoint and enables mutant H-Ras-driven cell transformation. Oncogene 28: 4469-4479.

Bergamaschi D, Samuels Y, O'Neil NJ, Trigiante G, Crook T, Hsieh JK, O'Connor DJ, Zhong S, Campargue I, Tomlinson $\mathrm{ML}$, et al. 2003. iASPP oncoprotein is a key inhibitor of p53 conserved from worm to human. Nat Genet 33: 162-167.

Chen CF, Yeh SH, Chen DS, Chen PJ, Jou YS. 2005. Molecular genetic evidence supporting a novel human hepatocellular carcinoma tumor suppressor locus at 13q12.11. Genes Chromosomes Cancer 44: 320-328.

Chiou SK, Hoa N. 2009. Up-regulation of GADD45 $\alpha$ expression by NSAIDs leads to apoptotic and necrotic colon cancer cell deaths. Apoptosis 14: 1341-1351.

Dong J, Feldmann G, Huang J, Wu S, Zhang N, Comerford SA, Gayyed MF, Anders RA, Maitra A, Pan D. 2007. Elucidation of a universal size-control mechanism in Drosophila and mammals. Cell 130: 1120-1133.

Espanel X, Sudol M. 2001. Yes-associated protein and p53-binding protein-2 interact through their WW and SH3 domains. I Biol Chem 276: 14514-14523.

Fernandez LA, Northcott PA, Dalton J, Fraga C, Ellison D, Angers S, Taylor MD, Kenney AM. 2009. YAP1 is amplified and up-regulated in hedgehog-associated medulloblastomas and mediates Sonic hedgehog-driven neural precursor proliferation. Genes Dev 23: 2729-2741.

Gorina S, Pavletich NP. 1996. Structure of the p53 tumor suppressor bound to the ankyrin and SH3 domains of 53BP2. Science 274: 1001-1005.

Jiang Z, Li X, Hu J, Zhou W, Jiang Y, Li G, Lu D. 2006. Promoter hypermethylation-mediated down-regulation of LATS1 and LATS2 in human astrocytoma. Neurosci Res 56: 450-458.

Jimenez-Velasco A, Roman-Gomez J, Agirre X, Barrios M, Navarro G, Vazquez I, Prosper F, Torres A, Heiniger A. 2005. Downregulation of the large tumor suppressor 2
(LATS2/KPM) gene is associated with poor prognosis in acute lymphoblastic leukemia. Leukemia 19: 2347-2350.

Kawahara M, Hori T, Chonabayashi K, Oka T, Sudol M, Uchiyama T. 2008. Kpm/Lats2 is linked to chemosensitivity of leukemic cells through the stabilization of p73. Blood 112: 3856-3866.

Labi V, Erlacher M, Kiessling S, Villunger A. 2006. BH3-only proteins in cell death initiation, malignant disease and anticancer therapy. Cell Death Differ 13: 1325-1338.

Lapi E, Di Agostino S, Donzelli S, Gal H, Domany E, Rechavi G, Pandolfi PP, Givol D, Strano S, Lu X, et al. 2008. PML, YAP, and $\mathrm{p} 73$ are components of a proapoptotic autoregulatory feedback loop. Mol Cell 32: 803-814.

Levy D, Adamovich Y, Reuven N, Shaul Y. 2007. The Yesassociated protein 1 stabilizes $\mathrm{p} 73$ by preventing Itch-mediated ubiquitination of p73. Cell Death Differ 14: 743-751.

Liu ZJ, Lu X, Zhang Y, Zhong S, Gu SZ, Zhang XB, Yang X, Xin HM. 2005. Downregulated mRNA expression of ASPP and the hypermethylation of the 5 '-untranslated region in cancer cell lines retaining wild-type p53. FEBS Lett 579: 15871590.

Liu X, Sempere LF, Ouyang H, Memoli VA, Andrew AS, Luo Y, Demidenko E, Korc M, Shi W, Preis M, et al. 2010. MicroRNA-31 functions as an oncogenic microRNA in mouse and human lung cancer cells by repressing specific tumor suppressors. J Clin Invest 120: 1298-1309.

Milyavsky M, Shats I, Erez N, Tang X, Senderovich S, Meerson A, Tabach Y, Goldfinger N, Ginsberg D, Harris CC, et al. 2003. Prolonged culture of telomerase-immortalized human fibroblasts leads to a premalignant phenotype. Cancer Res 63: 7147-7157.

Minsky N, Oren M. 2004. The RING domain of Mdm2 mediates histone ubiquitylation and transcriptional repression. Mol Cell 16: 631-639.

Oka T, Sudol M. 2009. Nuclear localization and pro-apoptotic signaling of YAP2 require intact PDZ-binding motif. Genes Cells 14: 607-615.

Overholtzer M, Zhang J, Smolen GA, Muir B, Li W, Sgroi DC, Deng CX, Brugge JS, Haber DA. 2006. Transforming properties of YAP, a candidate oncogene on the chromosome 11q22 amplicon. Proc Natl Acad Sci 103: 12405-12410.

Patel S, George R, Autore F, Fraternali F, Ladbury JE, Nikolova PV. 2008. Molecular interactions of ASPP1 and ASPP2 with the p53 protein family and the apoptotic promoters PUMA and Bax. Nucleic Acids Res 36: 5139-5151.

Powzaniuk M, McElwee-Witmer S, Vogel RL, Hayami T, Rutledge SJ, Chen F, Harada S, Schmidt A, Rodan GA, Freedman LP, et al. 2004. The LATS2/KPM tumor suppressor is a negative regulator of the androgen receptor. Mol Endocrinol 18: 2011-2023.

Samuels-Lev Y, O'Connor DJ, Bergamaschi D, Trigiante G, Hsieh JK, Zhong S, Campargue I, Naumovski L, Crook T, Lu X. 2001. ASPP proteins specifically stimulate the apoptotic function of p53. Mol Cell 8: 781-794.

Schilling T, Schleithoff ES, Kairat A, Melino G, Stremmel W, Oren M, Krammer PH, Muller M. 2009. Active transcription of the human FAS/CD95/TNFRSF6 gene involves the p53 family. Biochem Biophys Res Commun 387: 399-404.

Steinhardt AA, Gayyed MF, Klein AP, Dong J, Maitra A, Pan D, Montgomery EA, Anders RA. 2008. Expression of Yes-associated protein in common solid tumors. Hum Pathol 39: 1582-1589.

Strano S, Munarriz E, Rossi M, Castagnoli L, Shaul Y, Sacchi A, Oren M, Sudol M, Cesareni G, Blandino G. 2001. Physical interaction with Yes-associated protein enhances p73 transcriptional activity. J Biol Chem 276: 15164-15173. 
Strazisar M, Mlakar V, Glavac D. 2009. LATS2 tumour specific mutations and down-regulation of the gene in non-small cell carcinoma. Lung Cancer 64: 257-262.

Sullivan A, Lu X. 2007. ASPP: A new family of oncogenes and tumour suppressor genes. Br J Cancer 96: 196-200.

Takahashi Y, Miyoshi Y, Takahata C, Irahara N, Taguchi T, Tamaki Y, Noguchi S. 2005. Down-regulation of LATS1 and LATS2 mRNA expression by promoter hypermethylation and its association with biologically aggressive phenotype in human breast cancers. Clin Cancer Res 11: 1380-1385.

Thornton JK, Dalgleish C, Venables JP, Sergeant KA, Ehrmann IE, Lu X, Saunders PT, Elliott DJ. 2006. The tumour-suppressor protein ASPP1 is nuclear in human germ cells and can modulate ratios of CD44 exon V5 spliced isoforms in vivo. Oncogene 25: 3104-3112.

Toji S, Yabuta N, Hosomi T, Nishihara S, Kobayashi T, Suzuki S, Tamai K, Nojima H. 2004. The centrosomal protein Lats2 is a phosphorylation target of Aurora-A kinase. Genes Cells 9: 383-397.

Tront JS, Hoffman B, Liebermann DA. 2006. Gadd45a suppresses Ras-driven mammary tumorigenesis by activation of c-Jun $\mathrm{NH} 2$-terminal kinase and p38 stress signaling resulting in apoptosis and senescence. Cancer Res 66: 8448-8454.

Vigneron AM, Ludwig RL, Vousden KH. 2010. Cytoplasmic ASPP1 inhibits apoptosis through the control of YAP. Genes Dev (this issue). doi: 10.1101/gad.1954310.

Voorhoeve PM, le Sage C, Schrier M, Gillis AJ, Stoop H, Nagel R, Liu YP, van Duijse J, Drost J, Griekspoor A, et al. 2006. A genetic screen implicates miRNA-372 and miRNA-373 as oncogenes in testicular germ cell tumors. Cell 124: 1169-1181.

Wang W, El-Deiry WS. 2008. Restoration of p53 to limit tumor growth. Curr Opin Oncol 20: 90-96.

Yang JP, Hori M, Sanda T, Okamoto T. 1999. Identification of a novel inhibitor of nuclear factor-кB, RelA-associated inhibitor. J Biol Chem 274: 15662-15670.

Zaidi SK, Sullivan AJ, Medina R, Ito Y, van Wijnen AJ, Stein JL, Lian JB, Stein GS. 2004. Tyrosine phosphorylation controls Runx2-mediated subnuclear targeting of YAP to repress transcription. EMBO J 23: 790-799.

Zhao B, Wei X, Li W, Udan RS, Yang Q, Kim J, Xie J, Ikenoue T, $\mathrm{Yu}$ J, Li L, et al. 2007. Inactivation of YAP oncoprotein by the Hippo pathway is involved in cell contact inhibition and tissue growth control. Genes Dev 21: 2747-2761.

Zhao B, Li L, Tumaneng K, Wang CY, Guan KL. 2010. A coordinated phosphorylation by Lats and CK1 regulates YAP stability through SCF( $\beta$-TRCP). Genes Dev 24: 72-85.

Zhao J, Wu G, Bu F, Lu B, Liang A, Cao L, Tong X, Lu X, Wu M, Guo Y. 2010. Epigenetic silence of ankyrin-repeat-containing, SH3-domain-containing, and proline-rich-region-containing protein 1 (ASPP1) and ASPP2 genes promotes tumor growth in hepatitis B virus-positive hepatocellular carcinoma. Hepatology 51: 142-153. 


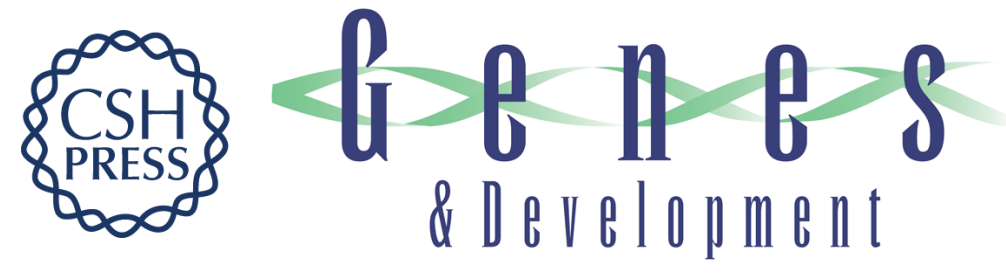

\section{The Lats2 tumor suppressor augments p53-mediated apoptosis by promoting the nuclear proapoptotic function of ASPP1}

Yael Aylon, Yaara Ofir-Rosenfeld, Norikazu Yabuta, et al.

Genes Dev. 2010, 24:

Access the most recent version at doi:10.1101/gad.1954410

\section{Supplemental http://genesdev.cshlp.org/content/suppl/2010/10/21/24.21.2420.DC1 Material}

Related Content Cytoplasmic ASPP1 inhibits apoptosis through the control of YAP Arnaud M. Vigneron, Robert L. Ludwig and Karen H. Vousden Genes Dev. November, 2010 24: 2430-2439

References This article cites 41 articles, 14 of which can be accessed free at: http://genesdev.cshlp.org/content/24/21/2420.full.html\#ref-list-1

Articles cited in:

http://genesdev.cshlp.org/content/24/21/2420.full.html\#related-urls

\section{License}

Email Alerting Receive free email alerts when new articles cite this article - sign up in the box at the top Service right corner of the article or click here.

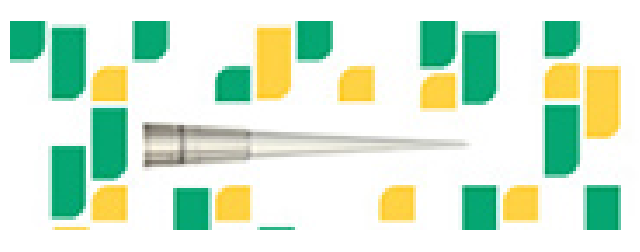

Focused on your science. 